\title{
Sesgo de deseabilidad social en medidas de valores organizacionales*
}

\section{Social desirability bias in measures of organizational values}

\author{
María Isabel de Campos ${ }^{\mathrm{a}}$ \\ Universidade São Francisco, Brasil \\ ORCID: http://orcid.org/0000-0002-5949-1583 \\ Fabián JaVier Marin Rueda \\ Universidade São Francisco, Brasil
}

a Autor de correspondencia. Correo electrónico:
isabel.playit@gmail.com

Para citar este artículo: Campos, M. I., \& Rueda, F. J. M. (2017). Sesgo de deseabilidad social en medidas de valores organizacionales. Universitas Psychologica, 16(2), 1-11.

https://doi.org/10.11144/Javeriana.upsy16-2.sdsm

\section{RESUMEN}

Este estudio tuvo como objetivo investigar la existencia de sesgo relacionado a la deseabilidad social en una investigación sobre los valores organizacionales. Participaron del estudio 232 personas que fueron divididas en dos grupos. El grupo 1, con 147 personas, fue formado por profesionales invitados a participar de la investigación por las empresas en las cuales trabajaban. Ellos fueron informados que la empresa recibiría un documento final con los resultados, sin ningún tipo de información individualizada. El grupo 2, con 85 personas, fue formado por profesionales invitados directamente por un investigador y fueron informados que en los resultados no habría ningún aspecto que permitiese su identificación, ni de su empresa. El instrumento aplicado fue el Inventario de Valores Organizacionales (IVO). Los resultados indicaron la presencia de un posible sesgo en las respuestas de los integrantes del grupo 1, apuntando para la necesidad de cuidados paliativos procesuales y estadísticos en situaciones semejantes de investigación en ambientes organizacionales.

Palabras clave

valores organizacionales; deseabilidad social; sesgo; comportamiento organizacional

\begin{abstract}
The present study aimed to investigate the existence of social desirability bias in research on organizational values. 232 people participated in the survey, divided into two groups. Group 1 was composed of professionals that were invited to respond to the survey by the companies they worked for, with 147 people. They were informed that the company would receive a consolidated report on the results, without any individualized information. Group 2, with 85 people, consisted of professionals invited directly by a researcher and they were informed that the results would not enable their identification, neither the company they worked for. The instrument used was the Organizational Values Inventory (IVO). The results indicated the presence of possible bias in the responses of members of group 1, pointing to the need for procedural and statistical palliative care when running similar assessments in organizational settings.
\end{abstract}

Keywords

Organizational Values; social desirability; bias; organizational behavior 


\section{Introducción}

En esta investigación, se analizó si existe diferencia en el modo de respuesta a una medida que evalúa el comportamiento organizacional dependiendo del origen de la invitación para tal, o sea, el motivo de la investigación fue verificar si invitados por la propia empresa, los encuestados presentan en sus respuestas efectos que pueden ser considerados como sesgo de deseabilidad social, fenómeno psicológico que se trata de medir con escalas desde 1960 (Gutiérrez, Sanz, Espinosa, Gesteira, \& García-Vera, 2016). El fenómeno hace que individuos se presenten a sí mismos o a sus organizaciones de una manera favorable. Este entendimiento al respecto de un posible sesgo es de gran importancia para los profesionales que actúan con psicología organizacional y del trabajo (POT) y con medidas del comportamiento organizacional (MCO), ya que los resultados encontrados pueden indicar que los procedimientos de medida y de evaluación necesitan considerar eventuales factores de influencia sobre las respuestas.

Para desarrollar este trabajo, la variable Diferente origen de las invitaciones para participar dividió a los investigados en dos grupos distintos. En el primer grupo, se incluyeron las personas que fueron invitadas por algún representante de la empresa (fundador, socio, presidente, director o gerente de $\mathrm{RH}$ ); y en el segundo grupo, se incluyeron las que fueron invitadas directamente por los investigadores o por otro conocido. Todos los individuos fueron informados que no serían identificados personalmente y tampoco la empresa en que trabajaban.

En las últimas décadas, profesionales de la POT y también de la administración han demostrado interés en estudios relativos a la evaluación psicológica en el contexto del trabajo. Pese a eso, una revisión sistemática realizada por Baptista, Rueda, Castro, Gomes y Silva (2011) en la base PePsic indicó que solo nueve artículos fueron publicados en el periodo de 2000 a 2009 relacionados con el grupo temático denominado por ellos de aspectos organizativos. Los autores observaron la existencia de una brecha en el tema de la evaluación psicológica en el contexto del trabajo.

Aun sobre el contexto del trabajo, los cambios veloces y constantes demandan, muchas veces, que procesos de mudanzas y transformaciones sean proyectados y ejecutados, con el objetivo de mantener la competitividad y el diferencial de la organización, por su bienestar y el de sus colaboradores (Castellanos \& Rojas, 2013; Denison, Hooijberg, Lane, \& Lief, 2012). Desde esta óptica, ampliamente defendida por Tamayo y Borges (2006), evaluar la percepción de los trabajadores sobre cuáles son los valores practicados por su organización y sobre cómo esos trabajadores se muestran satisfechos/insatisfechos con ellos mismos puede ofrecer subsidios y soporte para establecer cambios en gestión, estrategias y relaciones entre las organizaciones, una vez que esos valores constituyen un componente importante de la identidad social de la organización. De la asociación entre entender la importancia de los cambios en el ámbito organizacional y la brecha existente con relación a estudios sobre evaluación psicológica en ese contexto, se entiende que los estudios relacionados a medidas de valores organizacionales podrán ayudar a cubrir parcialmente la brecha señalada por Baptista et al. (2011).

Para que ese trabajo de medición sea efectivo, uno de los puntos de atención constante en el ámbito de las MCO es la calidad de estas medidas. Pese a eso, ese es un tema comúnmente citado como una de las principales deficiencias de las investigaciones del área, pues la mayor parte de las veces los investigadores basan sus conclusiones en instrumentos de autoinforme, no por ser mejores, sino por ser de fácil uso y, muchas veces, ser el único método viable de aplicación para medir constructos de interés (Donaldson \& Grant-Vallone, 2002). Pese a poder encontrar algunos ejemplos contrarios, como las investigaciones de Fernández-Arata y Merino-Soto (2016) y de Velo (2005), el gran problema de utilizar esos instrumentos de autoinforme es que en la mayoría de los casos no se considera el sesgo de deseabilidad social (Thompson \& Phua, 2005) o, en 
general, la variable común al método (Podsakoff, MacKenzie, Lee, \& Podsakoff, 2003).

Entre las diferentes MCO evaluadas, preocupaciones relativas a la calidad de las medidas, incluyendo el riesgo de la existencia de sesgos, pueden recaer sobre la percepción y la satisfacción indicada por los colaboradores de la organización en relación con los valores organizacionales practicados. Los valores organizacionales son definidos por Tamayo y Borges (2006) como principios o creencias relativas a los objetivos organizacionales deseables, que orientan la vida de la empresa y están al servicio de intereses individuales, colectivos o mixtos. Esos valores son un componente importante de la identidad organizacional, y es por medio de ellos que una empresa se diferencia de las demás en la sociedad y en el mercado. Esa diferencia es resultado, principalmente, de la jerarquía dada a esos valores, que incluye una preferencia y una distinción por parte de la organización, entre lo que es importante y secundario (Tamayo, Mendes, \& Paz, 2000).

El papel de los valores organizacionales es múltiple, tanto en las organizaciones privadas como en el sector público (Gabel-Shemueli, Yamada, \& Dolan, 2013). Además de crear y mantener la identidad de la organización, ellos sirven para guiar la vida organizacional, motivar sus miembros y, particularmente, para definir un contexto favorable al trabajo productivo y a la vida en la organización, tanto individual como colectiva (Tamayo, 2005).

Schwartz (1999) defiende que las dimensiones de valores culturales son más adecuadas que las personales para que se pueda comprender cómo los valores influyen en el contexto del trabajo, y destaca que esas dimensiones reflejan las preguntas básicas con las cuales sociedades o grupos sociales - lo cual incluye organizaciones - se deben confrontar a fin de regular la actividad de las personas. Los miembros de esos grupos, especialmente los responsables por tomar decisiones, reconocen y comunican esas preguntas/problemas, planifican respuestas y motivan unos a otros a enfrentarse con ellas. Por tanto, los valores son el vocabulario que aclara los objetivos socialmente aprobados y son usados para motivar la acción y para expresar y justificar las soluciones elegidas.

Schwartz (1999) también define que hay tres dimensiones de valores culturales y que esas dimensiones son bipolares, con siete tipos distintos de valores. Esa definición abarca la dimensión 1, que es la cuestión básica de la naturaleza de las relaciones entre el individuo y el grupo social y que presenta la bipolaridad (tipos de valores) autonomía y conservadurismo; la dimensión 2, que representa la garantía del comportamiento responsable que asegura el tejido social y que presenta la bipolaridad jerárquica y el igualitarismo; y la dimensión 3, que representa la relación humana con el mundo natural y social y que presenta la bipolaridad dominio y armonía. El séptimo tipo de valores es una subdivisión del tipo autonomía, que para Schwartz (1999) es compuesto por autonomía intelectual y autonomía emocional.

Es en ese contexto de los valores organizacionales y de la satisfacción de los funcionarios con esos valores que se delineó este trabajo. El objetivo es investigar la existencia de sesgos de deseabilidad social en las respuestas dadas por los participantes al responder un instrumento de autoinforme. El estudio sobre la existencia de sesgos en ese ámbito es un tema bastante importante, ya que los instrumentos de autoinforme continúan siendo los más utilizados para obtener resultados sobre las medidas del comportamiento organizacional (Thompson \& Phua, 2005), y en la mayoría de los casos son utilizados como única fuente de información, a pesar de la cantidad de estudios ya realizados sobre cuestiones referentes a la variabilidad común del método (Podsakoff et al, 2003). Además de eso, es importante destacar lo expuesto por Donaldson y Grant-Vallone (2002), al afirmar que la realización de estudios que verifiquen la existencia de sesgos sigue siendo un aspecto ignorado por la investigación en administración y psicología. 


\section{Método}

\section{Participantes}

Participaron 232 personas con edades entre 16 y 71 años $(M=33.49, D T=11.02)$ siendo que, de acuerdo con los grupos 1 y 2 anteriormente descriptos, 147 personas formaron el grupo $1 \mathrm{y}$ 85 el grupo 2. Con relación a la escolaridad, 48.7 \% tenía posgrado completo o incompleto, $46.1 \%$ tenía grado completo o incompleto y $5.2 \%$ tenía las enseñanzas primaria y secundaria completa o incompleta. El tiempo de trabajo en la organización estuvo entre 1 y 31 años. La Tabla 1 presenta un panorama general de otros datos de la muestra.

\section{TABLA 1}

Datos relativos a la muestra por sexo

\begin{tabular}{|c|c|c|c|c|c|c|c|c|c|c|c|c|c|c|}
\hline \multicolumn{3}{|c|}{ Participantes } & \multicolumn{4}{|c|}{ Funcionarios directos } & \multicolumn{8}{|c|}{ Cargos } \\
\hline & & & $\mathrm{Si}$ & & No & & $P / V I$ & & Geren & & $S / C$ & & Otros & \\
\hline & Frec & $\%$ & Frec & $\%$ & Frec & $\%$ & Frec & $\%$ & Frec & $\%$ & Frec & $\%$ & Frec & $\%$ \\
\hline Total & 232 & 100 & 104 & 45 & 128 & 55 & 19 & 8.2 & 33 & 14 & 41 & 18 & 139 & 60 \\
\hline M & 128 & 55 & 61 & 48 & 67 & 52 & 18 & 14 & 26 & 20 & 22 & 17 & 62 & 48 \\
\hline F & 104 & 45 & 37 & 36 & 67 & 64 & 1 & 1 & 7 & 6.7 & 19 & 18 & 77 & 74 \\
\hline G1 & 147 & 100 & 63 & 43 & 84 & 57 & 13 & 9 & 17 & 12 & 21 & 14 & 96 & 65 \\
\hline M & 81 & 55 & 40 & 49 & 41 & 51 & 13 & 16 & 14 & 17 & 11 & 14 & 43 & 53 \\
\hline $\mathrm{F}$ & 66 & 45 & 23 & 35 & 43 & 65 & 0 & 0 & 3 & 4.5 & 10 & 15 & 53 & 80 \\
\hline G2 & 85 & 100 & 41 & 48 & 44 & 52 & 6 & 7 & 16 & 18.8 & 20 & 24 & 43 & 51 \\
\hline M & 47 & 55 & 27 & 57 & 20 & 43 & 5 & 11 & 12 & 26 & 11 & 23 & 19 & 40 \\
\hline $\mathrm{F}$ & 38 & 45 & 14 & 37 & 24 & 63 & 1 & 2.6 & 4 & 11 & 9 & 24 & 24 & 63 \\
\hline
\end{tabular}

Fuente: elaboración propia.

Como se puede observar en la Tabla 1, del total de participantes, $55.2 \%$ era del sexo masculino. En relación con la posición ocupada en las organizaciones, $8.2 \%$ de los investigados indicó ocupar cargo de presidencia, vicepresidencia o directorio (13 personas en el grupo 1 y 6 personas del grupo 2), $14.2 \%$ ocupaban cargos de gerencia (17 personas del grupo 1 y 16 personas del 2), 17.7 \% de supervisión o coordinación y 59.9 \% dijo actuar en otros cargos (96 personas en el grupo 1 y 43 personas del grupo 2), siendo que del total, $55.2 \%$ dijo no tener subordinados directos (84 personas en el grupo 1 y 44 personas del grupo 2).

\section{Instrumento}

Inventario de Valores Organizacionales (IVO)

La elección del IVO (Tamayo et al., 2000) se dio por tres razones, (1) haber sido desarrollado con base en la teoría de los valores culturales de Schwartz, (2) las consideraciones ya mencionadas de ese autor sobre la teoría de los valores culturales ser la más adecuada cuando se trata de evaluar el contexto del trabajo y, principalmente, (3) porque esta escala permite medir no solo los valores organizacionales, sino también la satisfacción/insatisfacción de los trabajadores con relación a esos valores.

El IVO mide los valores organizacionales y es postulado sobre tres dimensiones bipolares formadas por los ejes, autonomía versus conservadurismo, jerarquía versus igualitarismo y dominio versus armonía. Permite medir los valores actualmente practicados por la organización de acuerdo con la percepción de sus funcionarios, solicitándoles que den puntaje entre 0 y 6 en relación con cuánto cada uno de los ítems presentados en la escala son importantes para la realidad actual de la organización. Permite también medir los valores en la forma "deseada" por los funcionarios, o sea, la percepción de ellos sobre cuánto cada ítem es importante para su organización, lo mismo que no ocurra. En ese caso, también es solicitado que atribuyan puntajes entre 0 y 6 .

Los 36 ítems del IVO corresponden a afirmaciones relacionadas con las dimensiones mencionadas. Ejemplos de ítems para cada uno de los polos son: Libertad para presentar sugerencias y opiniones sobre el trabajo para el polo Autonomía; Clima de relacionamiento amistoso entre los empleados para Conservadurismo; Tradición de respeto a las órdenes para Jerarquía; Tratamiento proporcional al mérito para Igualitarismo; Conquista de clientes en relación a la concurrencia para Dominio y Búsqueda del mejor precio en el mercado para Armonía.

Por el IVO también es posible calcular el Índice de Satisfacción con los Valores Organizacionales (ISVO) actualmente practicados en la empresa. 
El ISVO es calculado a través de los promedios entre las diferencias absoluta [1] entre la puntuación dada a los valores reales y a los valores deseados de cada factor. Corresponde aclarar que cuanto menor es la diferencia encontrada, mayor es la satisfacción con relación a los valores, una vez que una diferencia entre real y deseado equivalente a 0 indica una satisfacción de $100 \%$ con relación al factor medido y una diferencia equivalente a 6 indica una satisfacción de $0 \%$.

\section{Procedimiento}

La recolección de datos fue realizada por medio de la web, utilizando una herramienta desarrollada ad hoc, por medio de una plataforma basada en el lenguaje Java. La base de datos utilizada internamente fue la PostgreeSQL (sistema libre de administración de base de datos) y el alojamiento del sitio fue hecho por un servidor con seguridad de acceso y con banco en sistema aislado.

En su primera imagen, la herramienta web presentaba un documento en el cual el participante consentía en participar de la investigación, que previamente había sido aprobada por un comité de ética. Después que el sujeto seleccionaba la opción de participar, el inventario era presentado para sus respuestas. Después la etapa de recolección, los datos fueron exportados para una base del SPSS (Statistical Package for Social Sciences, versión 20), sobre la cual se generaron los puntajes de los valores organizacionales reales y deseados y los puntajes relativos a la satisfacción/ insatisfacción con los valores organizacionales. También fueron calculados otros resultados por medio de estadísticos descriptivos e inferenciales.

\section{Resultados}

Con el objetivo de verificar la existencia de diferencias significativas entre los resultados presentados por los participantes del grupo 1 y del grupo 2, lo que podría llevar a indicios de sesgos, fueron calculados los promedios para los valores reales, los valores deseados y el índice de satisfacción con los valores (reales). Para eso se procedió al test $t$ de Student, con nivel de significancia igual a 0.05 y se utilizó el procedimiento de Bonferroni $(\operatorname{con} p=0.05 / \mathrm{N}=$ 18), lo cual resultó en un nuevo valor aceptable de $p=0.003$. Los resultados son presentados en la Tabla 2.

\section{TABLA 2}

Promedios, DT y valores de $t$ y de p para los valores reales, valores deseados e ISVO de los grupos 1

(Empresa) y 2 (Directo)

\begin{tabular}{|c|c|c|c|c|c|c|c|}
\hline & & \multicolumn{2}{|c|}{$\overline{\text { Empresa }}$} & \multicolumn{2}{|c|}{ Directo } & \multirow[b]{2}{*}{$t$} & \multirow[b]{2}{*}{$p$} \\
\hline & & $\bar{P}$ & DT & $P$ & DT & & \\
\hline \multirow{6}{*}{ 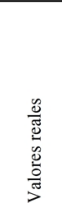 } & Autonomía & 3.86 & 1.23 & 3.27 & 1.23 & 3.52 & 0.001 \\
\hline & Conservadurismo & 4.18 & 1.01 & 3.66 & 1.05 & 3.69 & 0 \\
\hline & Jerarquía & 3.91 & 1.02 & 3.59 & 1.11 & 2.21 & 0.028 \\
\hline & Igualitarismo & 3.52 & 1.14 & 2.88 & 1.20 & 4.04 & 0 \\
\hline & Dominio & 3.84 & 1.03 & 3.50 & 0.98 & 2.49 & 0.013 \\
\hline & Armonía & 3.48 & 1.25 & 3.34 & 1.44 & 0.82 & 0.413 \\
\hline \multirow{6}{*}{ 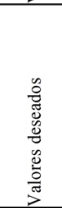 } & Autonomía & 5.26 & 0.64 & 5.25 & 0.63 & 0.12 & 0.906 \\
\hline & Conservadurismo & 5.29 & 0.59 & 5.40 & 0.57 & -1.41 & 0.161 \\
\hline & Jerarquía & 4.77 & 1.08 & 5.02 & 0.69 & -1.89 & 0.061 \\
\hline & Igualitarismo & 5 & 0.74 & 5 & 0.84 & -0.01 & 0.989 \\
\hline & Dominio & 4.86 & 0.84 & 4.78 & 0.79 & 0.70 & 0.486 \\
\hline & Armonía & 4.74 & 1.14 & 4.84 & 1.19 & -0.57 & 0.568 \\
\hline \multirow{6}{*}{ 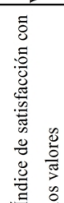 } & Autonomía & 1.44 & 0.98 & 2.01 & 1.15 & -4.04 & 0 \\
\hline & Conservadurismo & 1.21 & 0.90 & 1.76 & 1.04 & -4.23 & 0 \\
\hline & Jerarquía & 1.10 & 0.87 & 1.68 & 1.10 & -4.43 & 0 \\
\hline & Igualitarismo & 1.54 & 1.06 & 2.15 & 1.27 & -3.88 & 0 \\
\hline & Dominio & 1.24 & 0.83 & 1.66 & 1.11 & -3.26 & 0.001 \\
\hline & Armonia & 1.43 & 1.15 & 1.70 & 1.24 & -1.70 & 0.090 \\
\hline
\end{tabular}

Fuente: elaboración propia.

Para los valores reales, fueron encontradas diferencias significativas en tres de los seis factores medidos: autonomía, conservadurismo e igualitarismo. Se observa que todas las puntuaciones encontradas para los valores reales fueron mayores para el grupo 1 cuando fueron comparadas con las puntuaciones del grupo 2 .

En relación con el factor autonomía, la diferencia significativa encontrada muestra que los participantes del grupo 1 presentaron una puntuación promedio superior al grupo 2, indicando que las empresas para las cuales trabajan valorizan el empleado como un individuo autónomo, capaz de perseguir intereses propios y de fijar metas personales compatibles con las metas y normas organizacionales, dando 
importancia a la creatividad y a la responsabilidad individual.

Lo mismo ocurrió en relación con el factor conservadurismo, que evalúa cuánto la organización valoriza el mantenimiento de costumbres y estructuras de poder, manteniendo el status quo y restringiendo comportamientos que perturben las normas y las tradiciones de la empresa, así como en el factor igualitarismo, que se refiere a la calidad de la convivencia fuera de la organización, proporcionada por características de la propia organización.

En relación con los valores deseados, no se encontró ninguna diferencia significativa entre los grupos, y para el índice de satisfacción con los valores organizacionales, se encontraron diferencias significativas entre los grupos en cinco de los seis factores medidos. La excepción se dio en el factor armonía. Además de eso, se observó que todos los promedios de puntos encontrados para el ISVO fueron menores para el grupo 1.

De esta forma, además de las diferencias en relación con los valores autonomía, conservadurismo e igualitarismo, cuyos significados fueron relatados en el caso de los valores reales, los participantes del grupo 1 demostraron estar más satisfechos con los valores organizacionales practicados por sus empresas también en lo que se refiere a otros dos factores (factor jerarquía y dominio).

Con eso, los participantes del grupo 1 también se muestran más satisfechos con relación a la forma como sus empresas valorizan la autoridad, el poder social, la influencia, la fiscalización y la supervisión (jerarquía), y en cuanto a cómo ellas valorizan la capacidad de control de los recursos materiales, del mercado, de la tecnología y el conocimiento en el área específica de actuación (dominio).

Ampliando los resultados, y con el objetivo de investigar la existencia de indicios de sesgos, no solamente de las divisiones grupales 1 y 2, sino también en otras circunstancias, se buscó entender cuál sería el resultado de la comparación realizada en otros tres agrupamientos. El primer agrupamiento se refiere al sexo $(N=128$ para los hombres y $N=104$ para las mujeres). Los resultados se pueden ver en la Tabla 3.

TABLA 3

Promedios, DT y valores de $t$ y de p para los valores reales, valores deseados e ISVO por sexo

\begin{tabular}{|c|c|c|c|c|c|c|c|}
\hline & & \multirow{2}{*}{\multicolumn{2}{|c|}{$\begin{array}{c}\text { Masculino } \\
(\mathrm{N}=\mathbf{1 2 8})\end{array}$}} & \multirow{2}{*}{\multicolumn{2}{|c|}{$\begin{array}{l}\text { Femenino } \\
(\mathrm{N}=104)\end{array}$}} & \multirow[b]{3}{*}{$T$} & \\
\hline & & & & & & & \multirow[b]{2}{*}{$p$} \\
\hline & & $P$ & $D T$ & $P$ & $D T$ & & \\
\hline \multirow{6}{*}{ 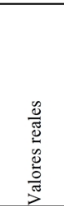 } & Autonomía & 3.82 & 1.23 & 3.42 & 1.27 & 2.41 & 0.017 \\
\hline & Conservadurismo & 4.07 & 0.98 & 3.89 & 1.13 & 1.35 & 0.179 \\
\hline & Jerarquía & 3.80 & 1.05 & 3.79 & 1.08 & 0.08 & 0.937 \\
\hline & Igualitarismo & 3.39 & 1.12 & 3.16 & 1.28 & 1.47 & 0.142 \\
\hline & Dominio & 3.67 & 0.95 & 3.78 & 1.11 & -0.79 & 0.433 \\
\hline & Armonía & 3.43 & 1.28 & 3.43 & 1.38 & 0.01 & 0.992 \\
\hline \multirow{6}{*}{ 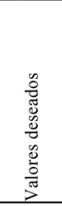 } & Autonomía & 5.28 & 0.66 & 5.23 & 0.60 & 0.61 & 0.543 \\
\hline & Conservadurismo & 5.30 & 0.63 & 5.38 & 0.52 & -1 & 0.316 \\
\hline & Jerarquía & 4.72 & 1.04 & 5.04 & 0.82 & -2.59 & 0.010 \\
\hline & Igualitarismo & 4.93 & 0.78 & 5.07 & 0.76 & -1.39 & 0.165 \\
\hline & Dominio & 4.79 & 0.82 & 4.88 & 0.83 & -0.82 & 0.412 \\
\hline & Armonía & 4.66 & 1.20 & 4.93 & 1.08 & -1.79 & 0.076 \\
\hline \multirow{6}{*}{ 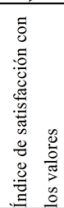 } & Autonomía & 1.49 & 1.06 & 1.83 & 1.08 & -2.41 & 0.017 \\
\hline & Conservadurismo & 1.31 & 0.87 & 1.54 & 1.11 & -1.74 & 0.083 \\
\hline & Jerarquía & 1.54 & 1.11 & 1.16 & 0.90 & -2.52 & 0.012 \\
\hline & Igualitarismo & 1.49 & 1.09 & 1.61 & 1 & -2.24 & 0.026 \\
\hline & Dominio & 1.36 & 0.87 & 1.44 & 1.07 & -0.65 & 0.519 \\
\hline & Armonía & 1.44 & 1.07 & 1.63 & 1.32 & -1.26 & 0.210 \\
\hline
\end{tabular}

Fuente: elaboración propia.

Al comparar los sexos no se encontraron diferencias significativas en los valores organizacionales reales. Lo mismo ocurrió con los valores deseados y con relación al ISVO. Esto indica que los hombres y las mujeres tienen percepciones semejantes sobre los valores de la organización en la que trabajan.

El segundo grupo analizado se refiere a funcionarios con cargos de confianza, o sea, funcionarios en la posición de presidente, vicepresidente, director(a), gerente, supervisor(a) o coordinador(a), con $N=93$, y los que ocupan otros tipos de posiciones, con $N=$ 139. Esos resultados pueden ser vistos en la Tabla 4. 
TABLA 4

Promedios, DT y valores de ty de p para los valores reales, valores deseados e ISVO por tipo de cargo

\begin{tabular}{|c|c|c|c|c|c|c|c|}
\hline & & \multirow{2}{*}{\multicolumn{2}{|c|}{$\begin{array}{c}\text { Cargo de } \\
\text { confianza } \\
(\mathrm{N}=93)\end{array}$}} & \multirow{2}{*}{\multicolumn{2}{|c|}{$\begin{array}{c}\text { Otros } \\
(\mathrm{N}=139)\end{array}$}} & \multirow[b]{3}{*}{$t$} & \\
\hline & & & & & & & \multirow[b]{2}{*}{$p$} \\
\hline & & $\bar{P}$ & $D T$ & $P$ & $D T$ & & \\
\hline \multirow{6}{*}{ 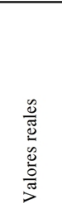 } & Autonomía & 3.60 & 1.14 & 3.67 & 1.34 & -0.37 & 0.713 \\
\hline & Conservadurismo & 3.96 & 0.88 & 4.01 & 1.15 & -0.34 & 0.738 \\
\hline & Jerarquía & 3.61 & 1.10 & 3.91 & 1.02 & -2.11 & 0.036 \\
\hline & Igualitarismo & 3.28 & 1.15 & 3.29 & 1.23 & -0.10 & 0.924 \\
\hline & Dominio & 3.76 & 0.99 & 3.69 & 1.05 & 0.50 & 0.615 \\
\hline & Armonía & 3.37 & 1.28 & 3.47 & 1.35 & -0.55 & 0.586 \\
\hline \multirow{6}{*}{ 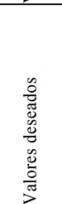 } & Autonomía & 5.24 & 0.56 & 5.26 & 0.68 & -0.23 & 0.816 \\
\hline & Conservadurismo & 5.37 & 0.51 & 5.31 & 0.62 & 0.81 & 0.421 \\
\hline & Jerarquía & 4.73 & 1 & 4.96 & 0.92 & -1.78 & 0.076 \\
\hline & Igualitarismo & 4.99 & 0.68 & 5 & 0.83 & -0.08 & 0.937 \\
\hline & Dominio & 4.88 & 0.71 & 4.80 & 0.89 & 0.76 & 0.451 \\
\hline & Armonía & 4.73 & 1.08 & 4.81 & 1.21 & -0.50 & 0.615 \\
\hline \multirow{6}{*}{ 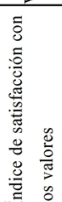 } & Autonomía & 1.67 & 0.97 & 1.63 & 1.15 & 0.23 & 0.817 \\
\hline & Conservadurismo & 1.48 & 0.81 & 1.37 & 1.09 & 0.80 & 0.426 \\
\hline & Jerarquía & 1.40 & 1.03 & 1.25 & 0.98 & 1.10 & 0.274 \\
\hline & Igualitarismo & 1.75 & 1.06 & 1.78 & 1.25 & -0.21 & 0.834 \\
\hline & Dominio & 1.46 & 1 & 1.35 & 0.94 & 0.91 & 0.364 \\
\hline & Armonía & 1.47 & 1.19 & 1.56 & 1.20 & -0.61 & 0.544 \\
\hline
\end{tabular}

Fuente: elaboración propia.

En el contexto de los valores reales, deseados y del ISVO, no se encontraron diferencias significativas entre los dos grupos. Esto indica que la percepción de los empleados con respecto a los valores de la organización es independiente de las posiciones mantenidas.

Finalizando, el tercer grupo estudiado se refiere a funcionarios con subordinados directos $(N=$ 104) y sin subordinados directos $(N=128)$. Los resultados pueden ser vistos en la Tabla 5 .

\section{TABLA 5}

Promedios, DT y valores de $t$ y de p para los valores reales, valores deseados e ISVO por jerarquía

\begin{tabular}{|c|c|c|c|c|c|c|c|}
\hline & & \multirow{2}{*}{\multicolumn{2}{|c|}{$\begin{array}{c}\text { Con } \\
\text { subordinados } \\
\text { directos } \\
(\mathrm{N}=104)\end{array}$}} & & & \\
\hline & & & & & $\begin{array}{l}\text { rdinado } \\
\text { ectos } \\
128 \text { ) }\end{array}$ & & \\
\hline & & $\bar{P}$ & $D T$ & $P$ & $D T$ & $t$ & $p$ \\
\hline \multirow{6}{*}{ 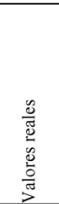 } & Autonomía & 3.76 & 1.21 & 3.54 & 1.30 & 1.30 & 0.193 \\
\hline & Conservadurismo & 3.90 & 0.99 & 4.06 & 1.10 & -1.20 & 0.233 \\
\hline & Jerarquía & 3.71 & 1.03 & 3.86 & 1.09 & -1.03 & 0.306 \\
\hline & Igualitarismo & 3.38 & 1.16 & 3.21 & 1.22 & 1.10 & 0.273 \\
\hline & Dominio & 3.78 & 0.98 & 3.66 & 1.06 & 0.91 & 0.361 \\
\hline & Armonía & 3.36 & 1.19 & 3.48 & 1.42 & -0.71 & 0.479 \\
\hline \multirow{6}{*}{ 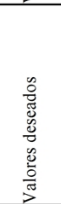 } & Autonomía & 5.30 & 0.60 & 5.22 & 0.66 & 0.95 & 0.341 \\
\hline & Conservadurismo & 5.35 & 0.54 & 5.32 & 0.61 & 0.46 & 0.648 \\
\hline & Jerarquía & 4.85 & 1 & 4.87 & 0.93 & -0.15 & 0.877 \\
\hline & Igualitarismo & 5.02 & 0.75 & 4.98 & 0.80 & 0.44 & 0.660 \\
\hline & Dominio & 4.88 & 0.74 & 4.79 & 0.88 & 0.86 & 0.390 \\
\hline & Armonía & 4.75 & 1.08 & 4.80 & 1.22 & -0.33 & 0.741 \\
\hline \multirow{6}{*}{ 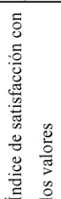 } & Autonomía & 1.57 & 1.04 & 1.71 & 1.11 & -0.94 & 0.346 \\
\hline & Conservadurismo & 1.51 & 0.94 & 1.33 & 1.02 & 1.41 & 0.159 \\
\hline & Jerarquía & 1.37 & 0.99 & 1.26 & 1.01 & 0.79 & 0.432 \\
\hline & Igualitarismo & 1.68 & 1.13 & 1.83 & 1.21 & -0.99 & 0.322 \\
\hline & Dominio & 1.38 & 0.95 & 1.40 & 0.97 & -0.11 & 0.913 \\
\hline & Armonía & 1.50 & 1.18 & 1.55 & 1.20 & -0.35 & 0.724 \\
\hline
\end{tabular}

Fuente: elaboración propia.

No se encontró ninguna diferencia significativa entre los dos grupos en el contexto de los valores reales, los valores deseados y el ISVO. Por tanto, la percepción de los valores de la organización también es similar entre grupos de personas que son responsables apenas por sus resultados individuales y de aquellos que también precisan responder por los resultados de otros empleados.

\section{Discusión}

Este estudio fue motivado porque en la literatura internacional se encuentran diversas menciones a la problemática basada en la existencia de sesgos de deseabilidad social al utilizar inventarios de autoinforme en investigaciones que se refieren al comportamiento organizacional (Donaldson \& Grant-Vallone, 2002; FernándezArata \& Merino-Soto, 2016; Podsakoff et al., 2003; Thompson \& Phua, 2005). En la literatura nacional brasileña. estos estudios todavía son bastante escasos. La intención fue contribuir a verificar la presencia de sesgos en una situación específica —invitación para participar en la investigación proveniente de distintas 
fuentes, siendo que una de esas fuentes podría ser generadora de sesgos- y también para estimular el interés por la realización de nuevas investigaciones sobre el tema.

La idea de que la empresa como fuente de invitación a la participación podría provocar un sesgo en las respuestas se basa en informaciones encontradas en la literatura. Entre los muchos motivos para la existencia de sesgos en cuestionarios de autoinforme, que van desde la variancia común del método hasta la necesidad de presentar una imagen más agradable que la real, Donaldson y Grant-Vallone (2002) señalan también el miedo, ya que los funcionarios que responden muchas veces piensan que existe por lo menos una posibilidad remota de que los empleadores tengan acceso a sus respuestas.

Por medio de la investigación realizada, fue posible verificar la presencia de sesgos de deseabilidad social en las respuestas dadas por los participantes del grupo 1 , aquellos que fueron invitados a participar de la investigación por alguien directamente ligado a la empresa. El sesgo puede ser observado, una vez que fueron encontradas diferencias significativas entre las puntuaciones medias presentadas por los dos grupos en análisis (grupo 1 y grupo 2) para tres de los factores evaluados en los valores reales (autonomía, conservadurismo e igualitarismo) y para cinco de los factores del ISVO. En el caso del ISVO, la excepción fue el factor armonía, para el cual apenas dos ítems del inventario son usados en el cálculo.

Esos resultados coinciden con lo señalado en la literatura (Gouveia, Guerra, Sousa, Santos, \& Costa, 2009; Thompson \& Phua, 2005; Podsakoff et al, 2003; Donaldson \& GrantVallone, 2002) e indican que los participantes del grupo 1 presentaron la tendencia de mostrar a las empresas en que trabajan los valores practicados por ellas y su satisfacción con esos valores de una manera favorable, independiente de sus sentimientos verdaderos acerca de esos temas. Esa presentación favorable puede ser afirmada con base en la puntuación media presentada en cada factor, una vez que la importancia dada por su empresa a los valores organizacionales y su nivel de satisfacción con esos valores se mostró superior, en todos los casos, a la puntuación dada por los participantes, cuya invitación a participar en la investigación no fue propuesta por la empresa (grupo control).

En los valores deseados, no fueron encontradas diferencias significativas entre las puntuaciones de los dos grupos. Esa ausencia puede ser explicada por el hecho de que lo que es deseado y particular no es comparable con aquello que se espera socialmente del individuo, y de esta forma, no se promueve la necesidad de la mejor presentación a los ojos de los investigadores o de la empresa.

Los resultados también permitieron observar que, cuando las respuestas fueron consideradas para los demás agrupamientos realizados, no se encontraron diferencias significativas. Particularmente para los grupos divididos por tipos de cargos ocupados, el hecho de no haber observado sesgos es un resultado opuesto a la visión presentada por Thompson y Phua (2005), que afirman que el sesgo de deseabilidad social es un aspecto importante y de preocupación de investigadores al realizar estudios con profesionales en puestos de confianza, una vez que esos profesionales tienen el deber fiduciario para presentar sus empresas, si no a sí mismos, de una manera favorable.

No fue encontrada ninguna diferencia significativa entre los grupos cuyos participantes tenían o no funcionarios subordinados. Esa ausencia parece indicar que las diferentes funciones y responsabilidades jerárquicas no influyen en la forma en cómo los funcionarios perciben los valores practicados por sus organizaciones, ni la forma como desean que sean practicados y la satisfacción con el status quo relacionado.

En un estudio desarrollado sobre los sesgos comunes a los métodos de investigación, Podsakoff et al. (2003) indicaron que las etapas del proceso de respuesta son la comprensión, la recuperación, la reflexión, la selección y el relato de la respuesta, y que el potencial sesgo de deseabilidad social está relacionado con la última etapa, en la cual el sujeto establece o da su respuesta en función de consistencia, de aceptabilidad o de otro criterio. Como 
recurso para minimizar varios tipos de sesgos en investigaciones, los autores sugieren que las mejores opciones son mantener y garantizar el anonimato de los participantes y estimular la sinceridad de las percepciones personales al responder.

Pese a eso, la realidad encontrada en este estudio no reflejó la visión de Podsakoff et al. (2003), ya que la garantía de anonimato y la solicitación por respuestas fieles al entendimiento personal no impidieron que los participantes del grupo 1 presentasen puntuaciones superiores a los del grupo 2, ofreciendo indicios de que en las empresas donde trabajan no solo existe una mayor valorización de los aspectos investigados por el instrumento, sino que ellos también se muestran más satisfechos con ese nivel de valorización que los participantes del grupo 2 . De esta forma, los resultados se aproximan más a la visión de Donaldson y Grant-Vallone (2002), que entienden que en investigaciones sobre comportamiento organizacional que usan el autoinforme el sesgo es más probable que ocurra, porque los funcionarios siempre creen que existe una posibilidad remota de que su empleador tenga acceso a sus respuestas.

Una forma común para el control de sesgos en investigaciones es el uso de escalas que miden la predisposición a la deseabilidad social, junto con escalas que miden los constructos de interés, como en Fernández-Arata y Merino-Soto (2016) y en Fonseca, Porto y Barroso (2012). Pese a eso, Thompson y Phua (2005) señalan la baja practicidad de esta forma de control en investigaciones sobre el comportamiento organizacional, no solo por la ampliación del número de ítems por ser respondidos, sino también por cuestiones conceptuales relativas a la calidad ambigua de los resultados obtenidos por estas escalas y por cuestiones prácticas relativas a la dificultad de obtener respuestas de hombres de negocios. En este último caso, afirman que la escala más utilizada en el contexto (la Escala Marlowe-Crowne) presenta ítems que pueden parecer triviales, complicados $\mathrm{O}$ irrelevantes para los profesionales que responden, lo cual puede comprometer el índice de respuesta de toda la investigación.
Independiente de los factores señalados, el investigador o el profesional organizacional brasileño tendría dificultades en aplicar tal escala en sus investigaciones, porque, hasta donde fue posible verificar, los estudios para validar un modelo local indican que cerca de $1 / 3$ de los ítems de la versión original no funcionaron adecuadamente en el contexto de la cultura local (Gouveia et al., 2009). Los referidos autores indican versiones reducidas como alternativas para llevar a cabo la medida, validando la versión $\mathrm{D}$ y la versión $\mathrm{C}$ de la escala original, respectivamente. Debemos destacar que para su uso en organizaciones de habla hispana también ya hay una versión reducida de la Escala de Marlowe-Crowne (Gutiérrez et al., 2016).

La falta de opciones de medidas puede ser una de las razones, por lo cual pocas referencias al fenómeno de deseabilidad social son realizadas en artículos que estudian medidas del comportamiento organizacional en Brasil. Cuando esas investigaciones son llevadas a cabo, la mención a la deseabilidad social acaba apareciendo apenas como una limitación del estudio (Gondim, Sobrinho, Santana, Santos, \& Saveia, 2013), o asociadas a la aplicación de técnicas estadísticas utilizadas como forma de control (Fonseca et al., 2012; Maurino \& De Domenico, 2012), o utilizando alguna escala que ya tenga incluidos ítems opuestos a la deseabilidad social (Borges, Argolo, \& Baker, 2006). Inclusive, la deseabilidad social aparece como una posibilidad declaradamente descartada, sin que sea realizado cualquier análisis que permita esa conclusión (Carvalho, Borges, \& Vikan, 2012).

Además de las dificultades inherentes al entorno y de la posible reducción de la calidad de los resultados en estudios realizados en el ámbito académico, el fenómeno del sesgo de deseabilidad social y las pocas opciones de control afectan también la credibilidad y el uso de escalas ya validadas junto a la práctica organizacional. Los valores organizacionales estudiados en este trabajo son apenas un ejemplo de constructo, cuya falta de precisión en las medidas puede provocar impactos indeseables en los procesos de 
gestión organizacional, tanto a nivel operativo como a nivel táctico y estratégico.

Los profesionales de recursos humanos, estrategas y gestores, así como los investigadores, demandan que la calidad y precisión de las medidas organizacionales sean reales. De otra forma, icómo se podrían tomar decisiones seguras y confiables en contextos organizacionales, con base en el resultado de instrumentos respondidos por los funcionarios de su organización?

A pesar de las imitaciones de este estudio, como el tamaño de la muestra, la utilización de herramienta web para la recolección de datos, lo que dificulta aclarar posibles dudas de los encuestados, y el levantamiento de un único constructo de interés, lo que impidió desarrollar más análisis que ampliasen la visión de los resultados, este estudio mostró que todavía no hay respuestas definitivas al último tema planteado. También mostró que la búsqueda por nuevas alternativas de control sobre el sesgo de deseabilidad social en MCO aún es necesaria, promoviendo y motivando la realización de nuevos estudios en este campo.

Mientras tanto, los investigadores y profesionales organizacionales deben tener conciencia de las cuestiones inherentes al sesgo en las investigaciones que utilizan instrumentos de autoinforme en las organizaciones. También es necesario buscar contornar la existencia de este sesgo utilizando paliativos de procedimiento y estadísticos ya recomendados por Podsakoff et al. (2003), que incluyen, entre otros, el delineamiento más cuidadoso del proyecto de medición, buscando obtener las medidas de variables predictoras y de criterio originarias de diferentes fuentes, la separación temporal de la medida de las variables predictoras y de criterio, la garantía antes mencionada del anonimato para reducir la aprensión al proporcionar respuestas, y por fin, el uso de varios procedimientos estadísticos que permiten controlar la variabilidad del método.

Con base en eso, se sugiere que sea realizado un nuevo estudio con el IVO, alternando la presentación de las preguntas a los participantes, separando la tabla de valores reales y valores deseados y tratando de entender si es la propia presentación del instrumento que promueve el sesgo. Otra posibilidad para futuras investigaciones sería considerar el desarrollo de un nuevo instrumento para medición de los valores organizacionales y de satisfacción de los colaboradores con relación a ellos, eventualmente basado en la misma teoría de valores de Schwartz (1999), pero que no sea de autoinforme. Podría ser desarrollado, por ejemplo, un Instrumento de Juicio Situacional (Ambiel, Campos, Alves, \& Silva, 2015) para la Evaluación de los Valores Organizacionales Practicados y Percibidos.

\section{Agradecimientos}

Nuestros agradecimentos a Coordenação de Aperfeiçoamento de Pessoal de Nível Superior (CAPES) por su apoyo.

\section{Referencias}

Ambiel, R. A. M., Campos, M. I. D., Alves, B. D. P., \& Silva, C. P. (2015). Fundamentos e aplicabilidade dos Testes de Julgamento Situacional no contexto da Psicologia Organizacional. Revista Psicologia Organizações e Trabalho, 15(3), 298-308.

Baptista, M. N., Rueda, F. J. M., Castro, N. R. D., Gomes, J. O., \& Silva, M. A. D. (2011). Análise de Artigos sobre Avaliação Psicológica no Contexto do Trabalho: Revisão Sistemática. Psicologia em Pesquisa, 5(2), 156-167.

Borges, L. D. O., Argolo, J. C. T., \& Baker, M. C. S. (2006). Os valores organizacionais e a síndrome de burnout: dois momentos em uma maternidade pública. Psicologia: Reflexão e Crítica, 19(1), 34-43.

Castellanos, C. R. R., \& Rojas, L. R. (2013). Relación entre cultura y valores organizacionales. Conciencia Tecnológica, $45,12-17$.

Carvalho, V., Borges, L. O., \& Vikan, A. (2012). Socialização organizacional: estudo comparativo entre servidores públicos 
brasileiros e noruegueses. Revista Eletrônica de Administração, 18(2), 339-371.

Denison, D., Hooijberg R., Lane N., \& Lief C. (2012). Leading culture change in global organizations: Aligning culture and strategy. San Francisco: Willey \& Sons.

Donaldson, S. I., \& Grant-Vallone, E. J. (2002). Understanding self-report bias in organizational behavior research. Journal of Business and Psychology, 17(2), 245-260.

Fernández-Arata, M., \& Merino-Soto, C. (2016). El lado socialmente deseable de las respuestas a medidas de burnout y engagement: un estudio preliminar. Revista Colombiana de Psicología, 25(1), 83-94.

Fonseca, A. M. O., Porto, J. B., \& Barroso, A. C. (2012). O efeito de valores pessoais nas atitudes perante estilos de liderança. Revista de Administração Mackenzie, 13(3), 122-149.

Gabel-Shemueli, R., Yamada, G., \& Dolan, S. (2013). Lo que vale el trabajo en el sector público: estudio exploratorio del significado de los valores organizacionales en el sector público en Perú. Revista de Psicología del Trabajo y de las Organizaciones, 29(2), 83-90.

Gondim, S. M. G., Sobrinho, A., Santana, V. S., Santos, V. M. D., \& Saveia, J. M. (2013). Gênero, autoconceito e trabalho na perspectiva de brasileiros e angolanos. Cadernos de Psicologia Social do Trabalho, 16(2), 153-165.

Gouveia, V. V., Guerra, V. M., Sousa, D. M. F. D., Santos, W. S., \& Costa, J. D. M. (2009). Escala de Desejabilidade Social de Marlowe-Crowne: evidências de sua validade fatorial e consistência interna. Avaliação Psicológica, 8(1), 87-98.

Gutiérrez, S., Sanz, J., Espinosa, R., Gesteira, C., \& García-Vera, M. P. (2016). La Escala de Deseabilidad Social de Marlowe-Crowne: baremos para la población general española y desarrollo de una versión breve. Anales de Psicología, 32(1), 206-217.

Maurino, S. V., \& De Domenico, S. M. R. (2012). Realização de valores pessoais no ambiente organizacional (RVP): olhando as relações entre indivíduo e organização para além dos valores pessoais. Revista de Administração Mackenzie, 13(3), 177-213.

Podsakoff, P. M., MacKenzie, S. B., Lee, J. Y., \& Podsakoff, N. P. (2003). Common method biases in behavioral research: A critical review of the literature and recommended remedies. Journal of Applied Psychology, 88(5), 879-903.

Schwartz, S. H. (1999). A theory of cultural values and some implications forwork. Applied Psychology: An International Review, 48(1), 23-47.

Tamayo, A. (2005). Valores Organizacionais e Comprometimento Afetivo. Revista de Administração Mackenzie, 3, 192-213

Tamayo, A., \& Borges, L. D. O. (2006). Valores do trabalho e das organizações. En M. Ros, \& V. V. Gouveia. Psicologia social dos valores humanos: desenvolvimentos teóricos, metodológicos e aplicados (pp. 397-431). São Paulo: Editora Senac.

Tamayo, A., Mendes A., \& Paz M. G. T. (2000). Inventário de Valores Organizacionais. Estudos de Psicologia, 5(2), 289-315.

Thompson, E. R., \& Phua, F. T. (2005). Reliability among senior managers of the Marlowe-Crowne short-form Social Desirability Scale. Journal of Business and Psychology, 19(4), 541-554.

Velo, J. S. (2005). Personalidad y deseabilidad social en contextos organizacionales: implicaciones para la práctica de la psicología del trabajo y las organizaciones. Papeles del Psicólogo, 92, 115-128.

\section{Notas}

* Artículo de investigación.

[1] Diferencia absoluta, o módulo, es una función algebraica que permite retirar la señal de un número real presentado siempre por medio de su valor absoluto. Se expresa como $|\mathrm{x}|$ y el resultado es que $\mid$ $\mathrm{x}|=|-\mathrm{x} \mid=\mathrm{x}$ 